\title{
ON THE CONVEXITY OF PIECEWISE-DEFINED FUNCTIONS *, **, ***
}

\author{
Heinz H. Bauschke ${ }^{1}$, Yves Lucet ${ }^{2}$ And Hung M. Phan ${ }^{3}$
}

\begin{abstract}
Functions that are piecewise defined are a common sight in mathematics while convexity is a property especially desired in optimization. Suppose now a piecewise-defined function is convex on each of its defining components - when can we conclude that the entire function is convex? In this paper we provide several convenient, verifiable conditions guaranteeing convexity (or the lack thereof). Several examples are presented to illustrate our results.
\end{abstract}

Mathematics Subject Classification. 26B25, 52A41, 65D17, 90C25.

Received August 21, 2014. Revised March 27, 2015

Published online April 27, 2016.

\section{INTRODUCTION}

Consider the function

$$
f: \mathbb{R}^{2} \rightarrow \mathbb{R}:(x, y) \mapsto \begin{cases}\frac{x^{2}+y^{2}+2 \max \{0, x y\}}{|x|+|y|}, & \text { if }(x, y) \neq(0,0) ; \\ 0, & \text { otherwise. }\end{cases}
$$

Keywords and phrases. Computer-aided convex analysis, convex function, convex interpolation, convex set, piecewise-defined function.

* HHB was supported by the Natural Sciences and Engineering Research Council (NSERC) of Canada under Discovery Grant \#216877-2013 and by the Canada Research Chair Program.

** YL was partially supported by NSERC Discovery grant \#298145-2013.

*** HMP was partially supported by NSERC Discovery grant program - accelerator supplement grant \#446219-2013 of HHB and an internal grant of University of Massachusetts Lowell.

1 Mathematics, University of British Columbia Okanagan, Kelowna, B.C. V1V 1V7, Canada. heinz.bauschke@ubc.ca

2 Computer Science, University of British Columbia Okanagan, Kelowna, B.C. V1V 1V7, Canada. yves.lucet@ubc.ca

3 Department of Mathematical Sciences, University of Massachusetts Lowell, Lowell, M.A. 01854, USA. hung_phan@uml.edu 
Clearly, $f$ is a piecewise-defined function with continuous components

$$
\begin{array}{llll}
f_{1}(x, y):=x+y & \text { on } & A_{1}:=\mathbb{R}_{+} \times \mathbb{R}_{+} ; \\
f_{2}(x, y):=\frac{x^{2}+y^{2}}{-x+y} & \text { on } & A_{2}:=\mathbb{R}_{-} \times \mathbb{R}_{+} ; \\
f_{3}(x, y):=x+y & \text { on } & A_{3}:=\mathbb{R}_{-} \times \mathbb{R}_{-} ; \\
f_{4}(x, y):=\frac{x^{2}+y^{2}}{x-y} & \text { on } & A_{4}:=\mathbb{R}_{+} \times \mathbb{R}_{-} .
\end{array}
$$

One may check that each $f_{i}$ is a convex function (see Example 6.1 below for details). However, whether or not $f$ itself is convex is not immediately clear. (As it turns out, $f$ is convex.)

On the other hand, if $f_{1}(x)=x$ on $A_{1}:=\mathbb{R}_{-}$and $f_{2}(x)=-x$ on $A_{2}:=\mathbb{R}_{+}$, then $f_{1}$ and $f_{2}$ are convex while the induced piecewise-defined function $f(x)=-|x|$ is not convex.

These and similar examples motivate the goal of this paper which is to present verifiable conditions guaranteeing the convexity of a piecewise-defined function provided that each component is convex. Special cases of our results have been known in the convex interpolation community (see Rem. 5.7). Moreover, our results have applications to computer-aided convex analysis (see Rem. 5.9).

(We mention in passing three different though related topics: The problem of guaranteeing the convexity of intersections of sublevel sets of functions was recently considered in [18]. Points of convexity, which localize the notion of convexity of a function, were investigated in $[9,11]$. In [12], it is shown that the convex envelope of a function arises as the solution of a nonlinear obstacle problem.)

The remainder of this paper is organized as follows. In Section 2, we collect various auxiliary results concerning convexity and differentiability. We also require properties of collections of sets and of functions which we develop in Sections 3 and 4, respectively. Our main results guaranteeing convexity are presented in Section 5. Various examples illustrating convexity and the lack thereof are discussed in Sections 6 and 7, respectively.

Notation: Throughout, $X$ is a Euclidean space with inner product $\langle\cdot, \cdot\rangle$ and induced norm $\|\cdot\| \cdot \mathbb{R}$ denotes the set of real numbers, $\mathbb{R}_{+}:=\{x \in \mathbb{R} \mid x \geq 0\}$, and $\mathbb{R}_{-}:=-\mathbb{R}_{+}$. For $x$ and $y$ in $X,[x, y]:=\{(1-t) x+t y \mid 0 \leq t \leq 1\}$ is the line segment connecting $x$ and $y$. Similarly, we set $] x, y[:=\{(1-t) x+t y \mid 0<t<1\},[x, y[:=$ $\{(1-t) x+t y \mid 0 \leq t<1\}$, and $] x, y]:=[y, x[$. For a subset $A$ of $X, \operatorname{conv} A, \operatorname{cl} A, \operatorname{int} A$, aff $A$, and $\operatorname{ri} A$ respectively denote the convex hull, the closure, the interior, the affine hull, and the relative interior of $A$. Furthermore, $\iota_{A}$ is the indicator function of $A$ defined by $\iota_{A}(x)=0$, if $x \in A$; and $+\infty$ otherwise. Let $f: X \rightarrow]-\infty,+\infty]=\mathbb{R} \cup\{+\infty\}$. The domain of $f$ is $\mathrm{D}_{f}:=\{x \in X \mid f(x)<+\infty\} ; f$ is said to be proper if $\mathrm{D}_{f} \neq \varnothing$. The restriction of $f$ on some subset $A$ of $X$ is denoted by $\left.f\right|_{A}$. A set-valued mapping $F$ from $X$ to another Euclidean space $Y$ is denoted by $F: X \rightrightarrows Y$; and its domain is $\mathrm{D}_{F}:=\{x \in X \mid F(x) \neq \varnothing\}$. For further background and notation, we refer the reader to $[1,10,13,14,19]$.

\section{Convexity AND DifFerentiability}

Let $f: X \rightarrow]-\infty,+\infty]$ be proper. For every $x \in X$, the subdifferential (in the sense of convex analysis) of $f$ at $x$, denoted by $\partial f(x)$, is the set of all vectors $x^{*} \in X$ such that

$$
(\forall y \in X) \quad\left\langle x^{*}, y-x\right\rangle \leq f(y)-f(x) .
$$

The induced operator $\partial f: X \rightrightarrows X$ has domain $\mathrm{D}_{\partial f}=\{x \in X \mid \partial f(x) \neq \varnothing\} \subseteq \mathrm{D}_{f}$.

Let us now present some auxiliary results concerning the convexity of a function.

Lemma 2.1. Let $f: X \rightarrow]-\infty,+\infty]$ and let $z_{1}$ and $z_{2}$ be in $\mathrm{D}_{f}$. Set $x:=(1-t) z_{1}+t z_{2}$, where $t \in[0,1]$, and assume that $x \in \mathrm{D}_{\partial f}$. Then $f(x) \leq(1-t) f\left(z_{1}\right)+t f\left(z_{2}\right)$. 
Proof. Let $x^{*} \in \partial f(x)$. Then $\left\langle x^{*}, z_{1}-x\right\rangle \leq f\left(z_{1}\right)-f(x)$ and $\left\langle x^{*}, z_{2}-x\right\rangle \leq f\left(z_{2}\right)-f(x)$. Hence

$$
\begin{aligned}
(1-t)\left\langle x^{*}, z_{1}-x\right\rangle & \leq(1-t)\left(f\left(z_{1}\right)-f(x)\right) ; \\
t\left\langle x^{*}, z_{2}-x\right\rangle & \leq t\left(f\left(z_{2}\right)-f(x)\right) .
\end{aligned}
$$

Adding up the last two inequalities, we obtain $0 \leq(1-t) f\left(z_{1}\right)+t f\left(z_{2}\right)-f(x)$.

Fact 2.2 (See [13], Thm. 6.1-6.3). Let $A$ be a nonempty convex subset of $X$. Then the following hold:

(1) $(\forall x \in \operatorname{cl} A)(\forall y \in \operatorname{ri} A)] x, y] \subseteq \operatorname{ri} A$.

(2) ri $A$ is nonempty and convex.

(3) $\operatorname{cl}(\operatorname{ri} A)=\operatorname{cl} A$.

Fact 2.3 (See [13], Thm. 23.4). Let $f: X \rightarrow]-\infty,+\infty]$ be convex and proper. Then ri $\mathrm{D}_{f} \subseteq \mathrm{D}_{\partial f}$.

Fact 2.4 (See [19], Thm. 2.4.1(iii)). Let $f: X \rightarrow]-\infty,+\infty]$ be proper. Assume that $\mathrm{D}_{f}=\mathrm{D}_{\partial f}$ is convex. Then $f$ is convex.

Proof. Take $z_{1}$ and $z_{2}$ in $\mathrm{D}_{f}$ and let $t \in[0,1]$. Set $x:=(1-t) z_{1}+t z_{2}$. Since $\partial f(x) \neq \varnothing$, Lemma 2.1 implies that $f(x) \leq(1-t) f\left(z_{1}\right)+t f\left(z_{2}\right)$. Therefore, $f$ is convex.

In the presence of continuity, Fact 2.4 admits the following extension.

Lemma 2.5. Let $f: X \rightarrow]-\infty,+\infty]$ be proper. Assume that $\mathrm{D}_{f}$ is convex, that $\left.f\right|_{\mathrm{D}_{f}}$ is continuous, and that ri $\mathrm{D}_{f} \subseteq \mathrm{D}_{\partial f}$. Then $f$ is convex.

Proof. Fact 2.2 implies that $\mathrm{riD}_{f}$ is convex and $\mathrm{cl}\left(\mathrm{riD}_{f}\right)=\operatorname{cl} \mathrm{D}_{f}$. Then the function $f+\iota_{\mathrm{riD}}$ is convex by Fact 2.4. Since $\left.f\right|_{\mathrm{D}_{f}}$ is continuous and ri $\mathrm{D}_{f}$ is dense in $\mathrm{D}_{f}$, we conclude that $\left.f\right|_{\mathrm{D}_{f}}$ is convex.

Given a nonempty subset $A$ of $X$, we define the dimension of $A$ to be the dimension of the linear subspace parallel to the affine hull of $A$, i.e., $\operatorname{dim} A:=\operatorname{dim}(\operatorname{aff} A-\operatorname{aff} A)$. We then have the following result.

Lemma 2.6. Let $f: X \rightarrow]-\infty,+\infty]$ be proper. Assume that $\left.f\right|_{\mathrm{D}_{f}}$ is continuous, that $\mathrm{D}_{f}$ is convex and at least 2-dimensional, and that there exists a finite subset $E$ of $X$ such that $\left.f\right|_{[x, y]}$ is convex for every segment $[x, y]$ contained in $\left(\mathrm{riD}_{f}\right) \backslash E$. Then $f$ is convex.

Proof. Take two distinct points $x$ and $y$ in $\mathrm{D}_{f}$, let $t \in[0,1]$, and set $z:=(1-t) x+t y$. Then $z \in \mathrm{D}_{f}$ because $\mathrm{D}_{f}$ is convex. It remains to show that

$$
f(z) \leq(1-t) f(x)+t f(y) .
$$

First, since $\mathrm{D}_{f}$ is convex and $\operatorname{dim}\left(\mathrm{D}_{f}\right) \geq 2$, there exists $w \in\left(\operatorname{ri} \mathrm{D}_{f}\right) \backslash \operatorname{aff}\{x, y\}$. For each $\left.\varepsilon \in\right] 0,1[$, set

$$
x_{\varepsilon}:=(1-\varepsilon) x+\varepsilon w, y_{\varepsilon}:=(1-\varepsilon) y+\varepsilon w, \text { and } z_{\varepsilon}:=(1-t) x_{\varepsilon}+t y_{\varepsilon} .
$$

Using Fact 2.2(1) and the finiteness of $E$, we have

$$
z_{\varepsilon} \in\left[x_{\varepsilon}, y_{\varepsilon}\right] \subseteq\left(\operatorname{riD}_{f}\right) \backslash E, \quad \text { for every } \varepsilon>0 \text { sufficiently small. }
$$

Because $f$ is convex on $\left[x_{\varepsilon}, y_{\varepsilon}\right]$, this implies

$$
f\left(z_{\varepsilon}\right) \leq(1-t) f\left(x_{\varepsilon}\right)+t f\left(y_{\varepsilon}\right) .
$$

Letting $\varepsilon \rightarrow 0^{+}$, we obtain (2.3) by using the continuity of $\left.f\right|_{\mathrm{D}_{f}}$. 
Remark 2.7 (The assumption on the dimension is important). Lemma 2.6 fails on $\mathbb{R}$ in the following sense. Consider $f: \mathbb{R} \rightarrow \mathbb{R}: x \mapsto-|x|$ and set $E:=\{0\}$. Then all assumptions of Lemma 2.6 hold except that $\mathrm{D}_{f}=\mathbb{R}$ is only 1-dimensional. Clearly, the conclusion of Lemma 2.6 is not true because $f$ is not convex.

We now turn our attention to differentiability properties. Recall that $f: X \rightarrow]-\infty,+\infty]$ is differentiable at $x \in$ int $\mathrm{D}_{f}$ if there exists $\nabla f(x) \in X$ such that $(\forall y \in X) f(y)-f(x)-\langle\nabla f(x), y-x\rangle=o(\|y-x\|) ; f$ is differentiable on subset $A$ of int $\mathrm{D}_{f}$ if $f$ is differentiable at every $x \in A$. We will require the following results.

Fact 2.8 (See [13], Thm. 25.1). Let $f: X \rightarrow]-\infty,+\infty]$ be convex and proper, and assume that $x \in \operatorname{int} \mathrm{D}_{f}$. Then $f$ is differentiable at $x$ if and only if $\partial f(x)$ is a singleton.

Fact 2.9 (See [13], Thm. 25.5). Let $f: X \rightarrow]-\infty,+\infty]$ be convex and proper, and let $\Omega$ be the set of points where $f$ is differentiable. Then $\Omega$ is a dense subset of int $\mathrm{D}_{f}$, and its complement in int $\mathrm{D}_{f}$ is a set of measure zero. Moreover, $\nabla f: \Omega \rightarrow X$ is continuous.

Fact 2.10 (See [13], Thm. 25.6). Let $f: X \rightarrow]-\infty,+\infty]$ be convex and proper such that $\mathrm{D}_{f}$ is closed with nonempty interior. Then

$$
\left(\forall x \in \mathrm{D}_{f}\right) \quad \partial f(x)=\operatorname{cl}(\operatorname{conv} S(x))+N_{\mathrm{D} f}(x),
$$

where $N_{\mathrm{D} f}(x):=\left\{x^{*} \in X \mid\left(\forall y \in \mathrm{D}_{f}\right)\left\langle x^{*}, y-x\right\rangle \leq 0\right\}$ is the normal cone to $\mathrm{D}_{f}$ at $x$ and $S(x)$ is the set of all limits of sequences $\left(\nabla f\left(x_{n}\right)\right)_{n \in \mathbb{N}}$ such that $f$ is differentiable at every $x_{n}$ and $x_{n} \rightarrow x$.

\section{Compatible systems of Sets}

In this section, we always assume that

$$
\begin{aligned}
& I \text { is a nonempty finite set; } \\
& \mathcal{A}:=\left\{A_{i}\right\}_{i \in I} \text { is a system of convex subsets of } X ; \\
& A:=\bigcup_{i \in I} A_{i} .
\end{aligned}
$$

Definition 3.1 (Compatible systems of sets). Assume (3.1). We say that $\mathcal{A}$ is a compatible system of sets if

$$
\left.\begin{array}{l}
i \in I \\
j \in I \\
i \neq j
\end{array}\right\} \Rightarrow \operatorname{cl} A_{i} \cap \operatorname{cl} A_{j} \cap \operatorname{ri} A=A_{i} \cap A_{j} \cap \operatorname{ri} A
$$

otherwise, we say that $\mathcal{A}$ is incompatible.

Example 3.2. Every system of finitely many closed convex subsets of $X$ is compatible.

Example 3.3 (Incompatible systems). Suppose that $X=\mathbb{R}^{2}$, that $I=\{1,2\}$, that $\left.\left.A_{1}=\right] 0,1\right] \times[0,1]$, and that $A_{2}=[-1,0] \times[0,1]$. Then $A=A_{1} \cup A_{2}=[-1,1] \times[0,1]$ and ri $\left.A=\right]-1,1[\times] 0,1\left[\right.$. Thus, $\mathcal{A}=\left\{A_{1}, A_{2}\right\}$ is incompatible because

$$
\left.\operatorname{cl} A_{1} \cap \operatorname{cl} A_{2} \cap \operatorname{ri} A=\{0\} \times\right] 0,1\left[\neq \varnothing=A_{1} \cap A_{2} \cap \operatorname{ri} A .\right.
$$

Definition 3.4 (Colinearly ordered tuple). The tuple of vectors $\left(x_{0}, \ldots, x_{n}\right) \in X^{n}$ is said to be colinearly ordered if the following hold:

(1) $\left[x_{0}, x_{n}\right]=\left[x_{0}, x_{1}\right] \cup \cdots \cup\left[x_{n-1}, x_{n}\right]$;

(2) $0 \leq\left\|x_{0}-x_{1}\right\| \leq\left\|x_{0}-x_{2}\right\| \leq \cdots \leq\left\|x_{0}-x_{n}\right\|$.

Proposition 3.5. Assume (3.1) and that $\mathcal{A}$ is a compatible system of sets (Def. 3.1). Then for every segment $[x, y]$ contained in ri $A$, there exists a colinearly ordered tuple $\left(x_{0}, \ldots, x_{n}\right)$ and $\left\{A_{i_{1}}, \ldots, A_{i_{n}}\right\} \subseteq \mathcal{A}$ such that

$$
x_{0}=x ; \quad x_{n}=y ; \quad \text { and } \quad(\forall k \in\{1, \ldots, n\}) \quad\left[x_{k-1}, x_{k}\right] \subseteq A_{i_{k}} .
$$


Proof. Let $[x, y] \subseteq \operatorname{ri} A$, with $x \in A_{i_{1}}$ for some $i_{1} \in I$. Set $x_{0}:=x$. For every $t \in[0,1]$, define

$$
x(t):=(1-t) x+t y .
$$

Furthermore, set

$$
t_{1}:=\sup \left\{t \in[0,1] \mid x(t) \in A_{i_{1}}\right\} \quad \text { and } \quad x_{1}:=x\left(t_{1}\right) .
$$

Then $\left[x_{0}, x_{1}\left[\subseteq A_{i_{1}}\right.\right.$ and $x_{1} \in \operatorname{cl} A_{i_{1}}$. Note also that $x_{1} \in \operatorname{ri} A$.

Case 1. $t_{1}=1$. Then $x_{1}=y \in \operatorname{cl} A_{i_{1}}$. Suppose that $y \notin A_{i_{1}}$. Then, $y \in A_{i_{2}}$ for some $i_{2} \in I$. It follows that $y \in \operatorname{cl} A_{i_{1}} \cap \operatorname{cl} A_{i_{2}} \cap \operatorname{ri} A=A_{i_{1}} \cap A_{i_{2}} \cap \mathrm{ri} A$, which is a contradiction. Therefore, $y \in A_{i_{1}}$ and we are done because $[x, y] \subseteq A_{i_{1}}$.

Case 2. $t_{1}<1$. Then there exist $\left.\left.\varepsilon \in\right] 0,1-t_{1}\right]$ and $A_{i_{2}} \in \mathcal{A} \backslash\left\{A_{i_{1}}\right\}$ such that

$$
] x_{1}, x_{2}\right] \subseteq A_{i_{2}} \quad \text { where } \quad x_{2}:=x\left(t_{1}+\varepsilon\right) .
$$

Hence $x_{1} \in \operatorname{cl} A_{i_{2}}$. We then have

$$
x_{1} \in \operatorname{cl} A_{i_{1}} \cap \operatorname{cl} A_{i_{2}} \cap \operatorname{ri} A=A_{i_{1}} \cap A_{i_{2}} \cap \operatorname{ri} A .
$$

So we have split $[x, y]$ into two line segments

$$
\left[x_{0}, x_{1}\right] \subseteq A_{i_{1}} \cap \operatorname{ri} A \quad \text { and } \quad\left[x_{1}, y\right] \subseteq\left(\bigcup_{i \in I \backslash\left\{i_{1}\right\}} A_{i}\right) \cap \operatorname{ri} A .
$$

Next, we repeat the above process for the segment $\left[x_{1}, y\right]$. Since $\mathcal{A}$ is finite, we eventually obtain (3.4).

Remark 3.6 (Closedness is not necessary for compatibility). We note that there are compatible systems of sets that are not closed. For example, suppose that $X=\mathbb{R}^{2}$, that $I=\{1,2\}$, that $A_{1}=[0,1[\times[0,1]$, and that $\left.\left.A_{2}=\right]-1,0\right] \times[0,1]$. Then neither $A_{1}$ nor $A_{2}$ is closed. However, since

$$
\operatorname{cl} A_{1} \cap \operatorname{cl} A_{2}=A_{1} \cap A_{2}=\{0\} \times[0,1],
$$

we deduce that $\mathcal{A}=\left\{A_{1}, A_{2}\right\}$ is compatible.

Definition 3.7 (Active index set). Assume (3.1). For every $x \in X$, we define the active index set associated with $\mathcal{A}$ by

$$
I_{\mathcal{A}}(x):=\left\{i \in I \mid x \in A_{i}\right\}
$$

and we will write $I(x)$ if there is no cause for confusion.

Proposition 3.8. Assume (3.1) and that $\mathcal{A}$ is a compatible system of sets (see Def. 3.1). Suppose that $x \in \operatorname{int} A$ and that $I_{\mathcal{A}}(x)=\{i\}$. Then $x \in \operatorname{int} A_{i}$.

Proof. Because int $A \neq \varnothing$, we have ri $A=\operatorname{int} A$. Suppose to the contrary that $x \notin \operatorname{int} A_{i}$. Then there exist $j \in I \backslash\{i\}$ and a sequence $\left(x_{n}\right)_{n \in \mathbb{N}}$ in $A_{j}$ such that that $x_{n} \rightarrow x$. It follows that

$$
x \in \operatorname{cl} A_{i} \cap \operatorname{cl} A_{j} \cap \operatorname{ri} A=A_{i} \cap A_{j} \cap \operatorname{ri} A,
$$

which is absurd because $I(x)=\{i\}$ by assumption. 


\section{Compatible systems of Functions}

In this section, we always assume that

$I$ is a nonempty finite set;

$\mathcal{F}:=\left\{f_{i}\right\}_{i \in I}$ is a system of proper convex functions from $X$ to $\left.]-\infty,+\infty\right]$;

$f:=\min _{i \in I} f_{i}$ is the piecewise-defined function associated with $\mathcal{F}$;

$I_{\mathcal{F}}: X \rightarrow I: x \mapsto\left\{i \in I \mid x \in \mathrm{D}_{f_{i}}\right\}$ is the active index set function.

We will write $I(x)$ instead of $I_{\mathcal{F}}(x)$ if there is no cause for confusion. Note that $\mathrm{D}_{f}=\bigcup_{i \in I} \mathrm{D}_{f_{i}}$.

Definition 4.1 (Compatible systems of functions). Assume (4.1). We say that $\mathcal{F}$ is a compatible system of functions if $\left.(\forall i \in I) f_{i}\right|_{D_{f_{i}}}$ is continuous and

$$
\left.\begin{array}{c}
i \in I \\
j \in I \\
i \neq j \\
\mathrm{D}_{f_{i}} \cap \mathrm{D}_{f_{j}} \neq \varnothing
\end{array}\right\}\left.\left.\Rightarrow f_{i}\right|_{\mathrm{D}_{f_{i}} \cap \mathrm{D}_{f_{j}}} \equiv f_{j}\right|_{\mathrm{D}_{f_{i}} \cap \mathrm{D}_{f_{j}}}
$$

We start with a useful lemma.

Lemma 4.2. Assume (4.1) and that $\mathcal{F}$ is compatible system of functions (Def. 4.1). Then

$$
(\forall x \in X) \quad \partial f(x) \subseteq \bigcap_{i \in I_{\mathcal{F}}(x)} \partial f_{i}(x)
$$

Proof. Suppose that $x^{*} \in \partial f(x)$ and that $i \in I_{\mathcal{F}}(x)$. Then $f_{i}(x)=f(x)$ and $(\forall y \in X) f_{i}(y)-f_{i}(x) \geq$ $f(y)-f(x) \geq\left\langle x^{*}, y-x\right\rangle$. Therefore, $x^{*} \in \partial f_{i}(x)$.

Lemma 4.3. Let $(a, b, c) \in X^{3}$ be colinearly ordered (Def. 3.4). Assume (4.1) with $I=\{1,2\}$, that $\mathcal{F}$ is compatible system of functions (Def. 4.1), that $\mathrm{D}_{f_{1}}=[a, b]$, that $\mathrm{D}_{f_{2}}=[b, c]$, and that

$$
\partial f_{1}(b) \cap \partial f_{2}(b) \neq \varnothing .
$$

Then $f$ is convex and

$$
\left(\forall x \in \mathrm{D}_{f}\right) \quad \partial f(x)=\bigcap_{i \in I_{\mathcal{F}}(x)} \partial f_{i}(x)= \begin{cases}\partial f_{1}(x), & \text { if } x \in[a, b[ \\ \partial f_{1}(b) \cap \partial f_{2}(b), & \text { if } x=b \\ \partial f_{2}(x), & \text { if } x \in] b, c]\end{cases}
$$

Proof. We assume that $a, b, c$ are pairwise distinct since the other cases are trivial. First, we show that

$$
\left(\forall x \in \left[a, b[) \quad \partial f_{1}(x) \subseteq \partial f(x) .\right.\right.
$$

Suppose that $x \in\left[a, b\left[\right.\right.$ and that $x^{*} \in \partial f_{1}(x)$. To establish (4.6), it suffices to show that

$$
(\forall y \in[a, c]) \quad\left\langle x^{*}, y-x\right\rangle \leq f(y)-f(x) .
$$


Indeed, (4.7) is true for $y \in[a, b]$ by definition of $\partial f_{1}(x)$ and $f$. Now suppose that $\left.\left.y \in\right] b, c\right]$. By (4.4), there exists $b^{*} \in \partial f_{1}(b) \cap \partial f_{2}(b)$. Then

$$
\begin{aligned}
\left\langle x^{*}, y-x\right\rangle & =\left\langle x^{*}, b-x\right\rangle+\left\langle x^{*}, y-b\right\rangle \\
& \leq f_{1}(b)-f_{1}(x)+\frac{\|y-b\|}{\|b-x\|}\left\langle x^{*}, b-x\right\rangle \\
& \leq f_{1}(b)-f_{1}(x)+\frac{\|y-b\|}{\|b-x\|}\left\langle b^{*}, b-x\right\rangle \\
& \leq f_{1}(b)-f_{1}(x)+\left\langle b^{*}, y-b\right\rangle \\
& \leq f_{1}(b)-f_{1}(x)+f_{2}(y)-f_{2}(b) \\
& =f_{2}(y)-f_{1}(x) \\
& =f(y)-f(x) .
\end{aligned}
$$

Hence (4.7) holds, as does (4.6).

Switching the roles of $f_{1}$ and $f_{2}$, we obtain analogously

$$
\left(\forall x \in \left[c, b[) \quad \partial f_{2}(x) \subseteq \partial f(x) .\right.\right.
$$

Next, it is straightforward to check that

$$
\partial f_{1}(b) \cap \partial f_{2}(b) \subseteq \partial f(b) .
$$

Since the reverse inclusions of (4.6), (4.9), and (4.10) follow from Lemma 4.2, we conclude that (4.5) holds. Using (4.4), (4.5) and Fact 2.3, we conclude that $\partial f(x) \neq \varnothing$ for all $x \in] a, c\left[=\operatorname{riD}_{f}\right.$. Finally, it follows from Lemma 2.5 that $f$ is convex.

Theorem 4.4. Let $\left(x_{0}, \ldots, x_{n}\right) \in X^{n+1}$ be colinearly ordered (Def. 3.4). Assume (4.1) with $I=\{1, \ldots, n\}$ and that $\mathcal{F}$ is a compatible system of functions (Def. 4.1) such that the following hold:

(1) $(\forall i \in\{1, \ldots, n\}) \mathrm{D}_{f_{i}}=\left[x_{i-1}, x_{i}\right]$.

(2) $(\forall i \in\{1, \ldots, n-1\}) \partial f_{i}\left(x_{i}\right) \cap \partial f_{i+1}\left(x_{i}\right) \neq \varnothing$.

Then $f$ is convex and

$$
\left(\forall x \in \mathrm{D}_{f}\right) \quad \partial f(x)=\bigcap_{i \in I_{\mathcal{F}}(x)} \partial f_{i}(x)
$$

Proof. If $n=1$, then the result is trivial. For $n \geq 2$, the result follows by inductively applying Lemma 4.3.

\section{MAIN RESULTS}

We are now ready for our main results.

Theorem 5.1 (Main result I). Assume (4.1), that $\mathcal{F}$ is a compatible system of functions (Def. 4.1), and that the following hold:

(a) $\mathrm{D}_{f}=\bigcup_{i \in I} \mathrm{D}_{f_{i}}$ is convex and at least 2-dimensional.

(b) $\left\{\mathrm{D}_{f_{i}}\right\}_{i \in I}$ is a compatible system of sets (Def. 3.1).

(c) There exists a finite subset $E$ of $X$ such that

$$
\left.\begin{array}{c}
x \in\left(\operatorname{riD}_{f}\right) \backslash E \\
\operatorname{card} I(x) \geq 2
\end{array}\right\} \Rightarrow \bigcap_{i \in I(x)} \partial f_{i}(x) \neq \varnothing .
$$


Then $f$ is convex and

$$
\left(\forall x \in \operatorname{riD} D_{f}\right) \quad \varnothing \neq \partial f(x) \subseteq \bigcap_{i \in I(x)} \partial f_{i}(x) .
$$

Proof. Let $[x, y] \subseteq\left(\mathrm{riD}_{f}\right) \backslash E$. By the compatibility in (b) and Proposition 3.5, there exist a colinearly ordered tuple $\left(x_{0}, \ldots, x_{n}\right) \in X^{n+1}$ and functions $f_{i_{1}}, \ldots, f_{i_{n}}$ in $\mathcal{F}$ such that

$$
x_{0}=x ; \quad x_{n}=y ; \quad \text { and } \quad(\forall k \in\{1, \ldots, n\})\left[x_{k-1}, x_{k}\right] \subseteq \mathrm{D}_{f_{i_{k}}} \cap \operatorname{ri} \mathrm{D}_{f} .
$$

Define

$$
(\forall k \in\{1, \ldots, n\}) \quad g_{k}:=f_{i_{k}}+\iota_{\left[x_{k-1}, x_{k}\right]}=f+\iota_{\left[x_{k-1}, x_{k}\right]} .
$$

Using (5.1), we see that, for every $k \in\{1, \ldots, n-1\}$,

$$
\partial g_{k}\left(x_{k}\right) \cap \partial g_{k+1}\left(x_{k}\right) \supseteq \partial f_{i_{k}}\left(x_{k}\right) \cap \partial f_{i_{k+1}}\left(x_{k}\right) \supseteq \bigcap_{i \in I\left(x_{k}\right)} \partial f_{i}\left(x_{k}\right) \neq \varnothing .
$$

By applying Theorem 4.4 to the system $\left\{g_{k}\right\}_{k \in\{1, \ldots, n\}}$, we see that $g=\min _{k \in\{1, \ldots, n\}} g_{k}=f+\iota_{[x, y]}$ is convex and hence so is $\left.f\right|_{[x, y]}$. In view of Lemma 2.6 , we obtain the convexity of $f$. Finally, for all $x \in \operatorname{ri} D_{f}$, we have $\partial f(x) \neq \varnothing$ by Fact 2.3. Therefore, (5.2) follows from Lemma 4.2.

Remark 5.2. We note an interesting feature in Theorem 5.1. In assumption (c), we require the non-emptiness of the subdifferential intersection (5.1) at all relative interior points except for finitely many points. Since our conclusion says that $f$ is convex, the subdifferential intersection is nonempty at every point in $\operatorname{ri}_{f}(\operatorname{see}$ Fact 2.3). That means, in order to check the convexity of $f$, we are allowed to ignore verifying (5.1) at finitely many points in ri $\mathrm{D}_{f}$. This turns out to be very convenient since checking (5.1) at certain points may not be obvious (see, for example, Example 6.1).

Remark 5.3 (Compatibility on the system of domains is essential). Theorem 5.1 fails if the domain compatibility assumption (b) is omitted: indeed, suppose that $X=\mathbb{R}^{2}$, that $I=\{1,2\}$, and that

$$
f_{1}=\iota_{[0,1] \times[0,1]} \text { and } f_{2}=\iota_{[-1,0[\times[0,1]}+1 .
$$

Then $\mathcal{F}$ is a compatible system of functions. Even though $\mathrm{D}_{f}=[-1,1] \times[0,1]$ is convex, $\left\{\mathrm{D}_{f_{i}} \cap \operatorname{ri} \mathrm{D}_{f}\right\}_{i \in I}$ is not a compatible system of sets. So, Theorem 5.1(b) is violated. Clearly, $f$ is not convex.

We thank a referee for a comment that caused us to point out the following result, which is complementary to Theorem 5.1. It deals with the case when $\mathrm{D}_{f}$ is 1-dimensional. For notational simplicity we state the result when $X=\mathbb{R}$.

Theorem 5.4. Assume that $X=\mathbb{R}$, that (4.1) holds, that $\mathcal{F}$ is a compatible system of functions (Def. 4.1), and that the following hold:

(a) $I=\{1,2, \ldots, n\}$ for some $n \geq 2$ and $(\forall i \in I) \operatorname{int} \mathrm{D}_{f_{i}} \neq \varnothing$.

(b) $(\forall i \in\{1, \ldots, n-1\}) x_{i}:=\max \mathrm{D}_{f_{i}}=\min _{f_{i+1}}$ and $\partial f_{i}\left(x_{i}\right) \cap \partial f_{i+1}\left(x_{i}\right) \neq \varnothing$.

(c) $\left\{\mathrm{D}_{f_{i}}\right\}_{i \in I}$ is a compatible system of sets (Def. 3.1).

Then $f$ is convex and $\left(\forall x \in \mathrm{D}_{f}\right) \partial f(x)=\bigcap_{i \in I_{\mathcal{F}}(x)} \partial f_{i}(x)$.

Proof. Take $x_{0} \in \mathrm{D}_{f_{1}}$ and $x_{n} \in \mathrm{D}_{f_{n}}$ such that $x_{0}<x_{1} \leq x_{n-1}<x_{n}$. By Theorem 4.4, the result holds for $f+\iota_{\left[x_{0}, x_{n}\right]}$. Since $x_{0}$ and $x_{n}$ were chosen arbitrarily, we are done.

Theorem 5.5 (Main result II). Assume (4.1), that $\mathcal{F}$ is a compatible system of functions (Def. 4.1), that each $f_{i}$ is differentiable on int $\mathrm{D}_{f_{i}} \neq \varnothing$, and that the following hold:

(a) $\mathrm{D}_{f}=\bigcup_{i \in I} \mathrm{D}_{f_{i}}$ is convex and at least 2-dimensional.

(b) $\left\{\mathrm{D}_{f_{i}}\right\}_{i \in I}$ is a compatible system of sets (Def. 3.1). 
(c) There exists a finite subset $E$ of $X$ such that

$$
\left.\begin{array}{c}
x \in\left(\operatorname{int} \mathrm{D}_{f}\right) \backslash E \\
\{i, j\} \subseteq I(x)
\end{array}\right\} \Rightarrow \quad \lim _{\substack{z \rightarrow x \\
z \in \operatorname{int} \mathrm{D}_{f_{i}}}} \nabla f_{i}(z)=\lim _{\substack{z \rightarrow x \\
z \in \operatorname{int} \mathrm{D}_{f_{j}}}} \nabla f_{j}(z) \quad \text { exists. }
$$

Then $f$ is convex; moreover, it is continuously differentiable on (int $\left.\mathrm{D}_{f}\right) \backslash E$.

Proof. We will prove the convexity of $f$ by using Theorem 5.1. Note that it suffices to verify assumption (c) of Theorem 5.1. To this end, let $x \in \operatorname{int}\left(\mathrm{D}_{f}\right) \backslash E$ such that $\operatorname{card} I(x) \geq 2$ and denote by $u_{x}^{*}$ the limit in (5.7). Fact 2.10 and Lemma 4.2 imply

$$
u_{x}^{*} \in \bigcap_{i \in I(x)} \partial f_{i}(x)
$$

So assumption (c) in Theorem 5.1 holds. Thus, we conclude that $f$ is convex.

Turning towards the differentiability statement, let $\Omega$ be the set of points at which $f$ is differentiable. Then $\bigcup_{i \in I}$ int $\mathrm{D}_{f_{i}} \subseteq \Omega \subseteq \operatorname{int} \mathrm{D}_{f}$.

Now let $x \in\left(\operatorname{int} D_{f}\right) \backslash E$. We consider two cases.

Case 1. Card $I(x)=1$. Then Proposition 3.8 implies that $x \in \operatorname{int} D_{f_{i}}$ for some $i \in I$, which implies that $x \in \Omega$.

Case 2. Card $I(x) \geq 2$. Since $x \in \operatorname{int} \mathrm{D}_{f}$, we obtain $N_{\mathrm{D}_{f}}(x)=\{0\}$. Hence, by Fact 2.10 , we have

$$
\partial f(x)=\operatorname{cl} \operatorname{conv}\left\{\lim _{n \in \mathbb{N}} \nabla f\left(z_{n}\right) \mid \Omega \ni z_{n} \rightarrow x\right\} .
$$

Let $\left(z_{n}\right)_{n \in \mathbb{N}}$ be a sequence in $\Omega$ such that $z_{n} \rightarrow x$, and let $\left(\varepsilon_{n}\right)_{n \in \mathbb{N}}$ be in $\mathbb{R}_{++}$such that $\varepsilon_{n} \rightarrow 0^{+}$. By Fact 2.9 , $\left.\nabla f\right|_{\Omega}$ is the continuous and because $\bigcup_{i \in I}$ int $\mathrm{D}_{f_{i}}$ is dense in $\mathrm{D}_{f}$, there exists a sequence $\left(y_{n}\right)_{n \in \mathbb{N}}$ in $\bigcup_{i \in I}$ int $D_{f_{i}}$ such that

$$
(\forall n \in \mathbb{N}) \quad\left\|y_{n}-z_{n}\right\| \leq \varepsilon_{n} \quad \text { and } \quad\left\|\nabla f\left(y_{n}\right)-\nabla f\left(z_{n}\right)\right\| \leq \varepsilon_{n} .
$$

Combining with (5.7), we deduce that

$$
y_{n} \rightarrow x \quad \text { and } \quad \lim _{n \in \mathbb{N}} \nabla f\left(z_{n}\right)=\lim _{n \in \mathbb{N}} \nabla f\left(y_{n}\right)=u_{x}^{*} .
$$

So, (5.9) becomes $\partial f(x)=\left\{u_{x}^{*}\right\}$. Thus, $f$ is differentiable at $x$ by Fact 2.8 .

Corollary 5.6. Assume (4.1), that $\mathcal{F}$ is a compatible system of functions (Def. 4.1), and that the following hold:

(a) $\mathrm{D}_{f}=\bigcup_{i \in I} \mathrm{D}_{f_{i}}$ is convex and at least 2-dimensional.

(b) $\left\{\mathrm{D}_{f_{i}}\right\}_{i \in I}$ is a compatible system of sets (Def. 3.1).

(c) $f$ is continuously differentiable on int $\mathrm{D}_{f}$.

Then $f$ is convex.

Proof. This follows from Theorem 5.5 with $E=\varnothing$.

Remark 5.7 (Convex interpolation). When $X=\mathbb{R}^{2}$, then Corollary 5.6 is known and of importance in the convex interpolation of data; (see, e.g., [5] Thm. 1, [6] Prop. 2.2, [15] Thm. 3.1, [2] Prop. 5.1 and the related [16] Thm. 3.6) for further details.

Corollary 5.8. Assume (4.1), that $\mathcal{F}$ is a compatible system of functions (Def. 4.1), that each $\mathrm{D}_{f_{i}}$ is closed, and that $(\forall i \in I)\left(\forall x \in \mathrm{D}_{f_{i}}\right) f_{i}(x)=\frac{1}{2}\left\langle x, A_{i} x\right\rangle+\left\langle b_{i}, x\right\rangle+\gamma_{i}$, where $A_{i}: X \rightarrow X$ is linear with $A_{i}=A_{i}^{*} \succeq 0$, $b_{i} \in X$, and $\gamma_{i} \in \mathbb{R}$. Furthermore, assume that $\mathrm{D}_{f}=\bigcup_{i \in I} \mathrm{D}_{f_{i}}$ is convex and at least 2-dimensional, and that

$$
\left.\begin{array}{c}
\{i, j\} \subseteq I \\
i \neq j \\
x \in \mathrm{D}_{f_{i}} \cap \mathrm{D}_{f_{j}}
\end{array}\right\} \Rightarrow A_{i} x+b_{i}=A_{j} x+b_{j} .
$$

Then $f$ is convex; moreover, it is continuously differentiable on int $\mathrm{D}_{f}$. 
Proof. This follows from Example 3.2 and Theorem 5.5 with $E=\varnothing$ since $\nabla f_{i}(x)=A_{i} x+b_{i}$ when $x \in \operatorname{int} \mathrm{D}_{f_{i}}$.

Remark 5.9 (Piecewise linear-quadratic function). Consider Corollary 5.8 with the additional assumption that each $\mathrm{D}_{f_{i}}$ is a polyhedral set. Then Corollary 5.8 provides a sufficient condition for checking the convexity of $f$ which in this case is a piecewise linear-quadratic function. These functions play a role in computer-aided convex analysis (see [7], [14] Sect. 10.E) and also [17] for further information on functions of this type). Moreover, we thus partially answer an open question from ([8], Sect. 23.4.2). Our results also enhance our understanding of how nonconvexity occurs and form another step towards building a nonconvex toolbox that extends current bivariate computational convex analysis algorithms $[3,4]$.

\section{Checking COnvexity}

We start with an application of Theorem 5.5.

Example 6.1. The function

$$
f: \mathbb{R}^{2} \rightarrow \mathbb{R}:(x, y) \mapsto\left\{\begin{array}{lll}
\frac{x^{2}+y^{2}+2 \max \{0, x y\}}{|x|+|y|}, & \text { if } \quad(x, y) \neq(0,0) ; \\
0, & \text { if } \quad(x, y)=(0,0)
\end{array}\right.
$$

is convex, and differentiable on $\mathbb{R}^{2} \backslash\{(0,0)\}$.

Proof. First, set $I:=\{1, \ldots, 4\}$ and

$$
\begin{aligned}
& f_{1}(x, y):= \begin{cases}x+y, & \text { if }(x, y) \in A_{1}:=\mathbb{R}_{+}^{2}, \\
+\infty, & \text { otherwise; }\end{cases} \\
& f_{2}(x, y):= \begin{cases}\frac{x^{2}+y^{2}}{-x+y}, & \text { if }(x, y) \in A_{2}:=\mathbb{R}_{-} \times \mathbb{R}_{+}, \\
+\infty, & \text { otherwise; }\end{cases} \\
& f_{3}(x, y):= \begin{cases}-x-y, & \text { if }(x, y) \in A_{3}:=\mathbb{R}_{-}^{2}, \\
+\infty, & \text { otherwise; }\end{cases} \\
& f_{4}(x, y):= \begin{cases}\frac{x^{2}+y^{2}}{x-y}, & \text { if }(x, y) \in A_{4}:=\mathbb{R}_{+} \times \mathbb{R}_{-}, \\
+\infty, & \text { otherwise. }\end{cases}
\end{aligned}
$$

Then $\left\{f_{i}\right\}_{i \in I}$ is a compatible system of functions (Def. 4.1) with $f$ being the corresponding piecewise-defined function. Moreover, $\left\{D_{f_{i}}\right\}_{i \in I}=\left\{A_{i}\right\}_{i \in I}$ is a compatible system of sets.

Each $f_{i}$ is differentiable on $\operatorname{int} A_{i}$ with the gradient given by

$$
\begin{aligned}
& \nabla f_{1}(x, y)=(1,1) \quad \text { for } \quad(x, y) \in \operatorname{int} A_{1} \\
& \nabla f_{2}(x, y)=\left(\frac{-x^{2}+2 x y+y^{2}}{(x-y)^{2}}, \frac{-x^{2}-2 x y+y^{2}}{(x-y)^{2}}\right) \text { for } \quad(x, y) \in \operatorname{int} A_{2} ; \\
& \nabla f_{3}(x, y)=(-1,-1) \quad \text { for } \quad(x, y) \in \operatorname{int} A_{3} ; \\
& \nabla f_{4}(x, y)=\left(\frac{x^{2}-2 x y-y^{2}}{(x-y)^{2}}, \frac{x^{2}+2 x y-y^{2}}{(x-y)^{2}}\right) \text { for } \quad(x, y) \in \operatorname{int} A_{4} .
\end{aligned}
$$

One readily checks that the Hessian of each $f_{i}$ is positive semi-definite on $\operatorname{int} A_{i}$; hence, by the continuity of $\left.f_{i}\right|_{\mathrm{D}_{f_{i}}}$, we have that $f_{i}$ is convex. 
Moreover,

$$
\begin{array}{lll}
(\forall a>0) & \lim _{\substack{(x, y) \rightarrow(a, 0) \\
(x, y) \in \operatorname{int} A_{1}}} \nabla f_{1}(x, y)=\lim _{\substack{(x, y) \rightarrow(a, 0) \\
(x, y) \in \operatorname{int} A_{4}}} \nabla f_{4}(x, y)=(1,1) ; \\
(\forall a<0) & \lim _{\substack{(x, y) \rightarrow(a, 0) \\
(x, y) \in \operatorname{int} A_{2}}} \nabla f_{2}(x, y)=\lim _{\substack{(x, y) \rightarrow(a, 0) \\
(x, y) \in \operatorname{int} A_{3}}} \nabla f_{3}(x, y)=(-1,-1) ; \\
(\forall b>0) \quad \lim _{\substack{(x, y) \rightarrow(0, b) \\
(x, y) \in \operatorname{int} A_{1}}} \nabla f_{1}(x, y)=\lim _{\substack{(x, y) \rightarrow(0, b) \\
(x, y) \in \operatorname{int} A_{2}}} \nabla f_{2}(x, y)=(1,1) ; \\
(\forall b<0) \quad \lim _{\substack{(x, y) \rightarrow(0, b) \\
(x, y) \in \operatorname{int} A_{3}}} \nabla f_{3}(x, y)=\lim _{\substack{(x, y) \rightarrow(0, b) \\
(x, y) \in \operatorname{int} A_{4}}} \nabla f_{4}(x, y)=(-1,-1) .
\end{array}
$$

Now set $E:=\{(0,0)\}$. From the above computations, we observe that all assumptions of Theorem 5.5 are satisfied. Thus, we conclude that $f$ is a convex function that is also continuously differentiable away from the origin.

In fact, the function defined by (6.1) is actually a norm since it is clearly positively homogeneous. An analogous use of Theorem 5.5 allows for a systematic proof of the convexity of the function considered next.

Example 6.2. The function

$$
f: \mathbb{R}^{2} \rightarrow \mathbb{R}:(x, y) \mapsto \begin{cases}\sqrt{x^{6}+y^{4}}, & \text { if } x y \geq 0 ; \\ |x|^{3}+y^{2}, & \text { otherwise }\end{cases}
$$

is a convex and continuously differentiable.

We conclude this section with an application of Theorem 5.1.

Example 6.3. The function

$$
f: \mathbb{R}^{2} \rightarrow \mathbb{R}:(x, y) \mapsto f(x, y):= \begin{cases}\sqrt{x^{4}+y^{2}}, & \text { if } \quad x y \geq 0 \\ x^{2}+|y|, & \text { otherwise }\end{cases}
$$

is convex.

Proof. First, set $I:=\{1, \ldots, 4\}$ and

$$
\begin{aligned}
f_{1}(x) & := \begin{cases}\sqrt{x_{1}^{4}+x_{2}^{2},}, & \text { if } x \in A_{1}:=\mathbb{R}_{+}^{2}, \\
+\infty, & \text { otherwise; }\end{cases} \\
f_{2}(x) & := \begin{cases}x_{1}^{2}+x_{2}, & \text { if } x \in A_{2}:=\mathbb{R}_{-} \times \mathbb{R}_{+}, \\
+\infty, & \text { otherwise; }\end{cases} \\
f_{3}(x): & = \begin{cases}\sqrt{x_{1}^{4}+x_{2}^{2}}, & \text { if } x \in A_{3}:=\mathbb{R}_{-}^{2}, \\
+\infty, & \text { otherwise; }\end{cases} \\
f_{4}(x): & = \begin{cases}x_{1}^{2}-x_{2}, & \text { if } x \in A_{4}:=\mathbb{R}_{+} \times \mathbb{R}_{-}, \\
+\infty, & \text { otherwise. }\end{cases}
\end{aligned}
$$

Then $\left\{f_{i}\right\}_{i \in I}$ is a compatible system of functions (Def. 4.1) with $f$ being the corresponding piecewise function. Moreover, $\left\{D_{f_{i}}\right\}_{i \in I}=\left\{A_{i}\right\}_{i \in I}$ is a compatible system of sets. 
Each $f_{i}$ is differentiable on int $A_{i}$ with the gradient given by

$$
\begin{aligned}
& \nabla f_{1}(x)=\left(\frac{2 x_{1}^{3}}{\sqrt{x_{1}^{4}+x_{2}^{2}}}, \frac{x_{2}}{\sqrt{x_{1}^{4}+x_{2}^{2}}}\right) \text { for } x \in \operatorname{int} A_{1} ; \\
& \nabla f_{2}(x)=\left(2 x_{1}, 1\right) \quad \text { for } \quad x \in \operatorname{int} A_{2} ; \\
& \nabla f_{3}(x)=\left(\frac{2 x_{1}^{3}}{\sqrt{x_{1}^{4}+x_{2}^{2}}}, \frac{x_{2}}{\sqrt{x_{1}^{4}+x_{2}^{2}}}\right) \text { for } \quad x \in \operatorname{int} A_{3} ; \\
& \nabla f_{4}(x)=\left(2 x_{1},-1\right) \quad \text { for } \quad x \in \operatorname{int} A_{4} .
\end{aligned}
$$

Next, since the Hessian of $f_{i}$ is positive semidefinite on $\operatorname{int} A_{i}$, we deduce that each $f_{i}$ is convex.

Now set $E:=\{(0,0)\}$. We will verify (5.1). Note that simple computations show the following:

For $x=\left(0, x_{2}\right) \in\left(A_{1} \cap A_{2}\right) \backslash E$,

$$
\lim _{\substack{z \rightarrow x \\ z \in \text { int } A_{1}}} \nabla f_{1}(z)=\lim _{\substack{z \rightarrow x \\ z \in \operatorname{int} A_{2}}} \nabla f_{2}(z)=(0,1) \in \partial f_{1}(x) \cap \partial f_{2}(x) .
$$

For $x=\left(0, x_{2}\right) \in\left(A_{3} \cap A_{4}\right) \backslash E$,

$$
\lim _{\substack{z \rightarrow x \\ z \in \operatorname{int} A_{3}}} \nabla f_{3}(z)=\lim _{\substack{z \rightarrow x \\ z \in \operatorname{int} A_{4}}} \nabla f_{4}(z)=(0,-1) \in \partial f_{3}(x) \cap \partial f_{4}(x) .
$$

For $x=\left(x_{1}, 0\right) \in\left(A_{2} \cap A_{3}\right) \backslash E$,

$$
\begin{aligned}
& \lim _{\substack{z \rightarrow x \\
z \in \text { int } A_{2}}} \nabla f_{2}(z)=\left(2 x_{1}, 1\right) \quad \text { and } \quad N_{A_{2}}(x)=\{0\} \times \mathbb{R}_{-} ; \\
& \lim _{z \rightarrow x} \nabla f_{3}(z)=\left(2 x_{1}, 0\right) \quad \text { and } \quad N_{A_{3}}(x)=\{0\} \times \mathbb{R}_{+} .
\end{aligned}
$$

Then, using Fact 2.10, we conclude that $\partial f_{2}(x) \cap \partial f_{3}(x) \neq \varnothing$.

For $x=\left(x_{1}, 0\right) \in\left(A_{1} \cap A_{4}\right) \backslash E$,

$$
\begin{gathered}
\lim _{\substack{z \rightarrow x \\
z \in \text { int } A_{1}}} \nabla f_{1}(z)=\left(2 x_{1}, 0\right) \quad \text { and } \quad N_{A_{1}}(x)=\{0\} \times \mathbb{R}_{-} ; \\
\lim _{\substack{z \rightarrow x \\
z \in \text { int } A_{4}}} \nabla f_{4}(z)=\left(2 x_{1},-1\right) \quad \text { and } \quad N_{A_{4}}(x)=\{0\} \times \mathbb{R}_{+} .
\end{gathered}
$$

Then, using Fact 2.10, we conclude that $\partial f_{1}(x) \cap \partial f_{4}(x) \neq \varnothing$.

So, we have verified that assumption (c) in Theorem 5.1 holds. Therefore, $f$ is convex by Theorem 5.1.

\section{Detecting the LACK OF CONVEXity}

The nonempty subdifferential intersection condition (5.1) is indeed crucial for the check of convexity: we will see in the following result that the violation of (5.1) leads to nonconvexity.

Theorem 7.1 (Detecting lack of convexity). Assume (4.1), that $\mathcal{F}$ is a compatible system of functions (Def. 4.1), and that

$$
\left(\exists x \in \operatorname{riD} D_{f}\right) \bigcap_{i \in I(x)} \partial f_{i}(x)=\varnothing .
$$

Then $f$ is not convex. 
Proof. Using Lemma 4.2, we have $\partial f(x) \subseteq \bigcap_{i \in I(x)} \partial f_{i}(x)=\varnothing$. Therefore, by Fact 2.3, $f$ is not convex.

Using Theorem 7.1, we will now illustrate that the finiteness assumption on $E$ is important for our main results (Thms. 5.1 and 5.5).

Example 7.2. Suppose that $X=\mathbb{R}^{2}$, set

$$
\begin{aligned}
f_{1}(x, y) & := \begin{cases}\max \{-x, y\}, & \text { if }(x, y) \in A_{1}:=\mathbb{R}_{+} \times \mathbb{R} \\
+\infty, & \text { otherwise }\end{cases} \\
f_{2}(x, y) & := \begin{cases}\max \{x, y\}, & \text { if } \quad(x, y) \in A_{2}:=\mathbb{R}_{-} \times \mathbb{R} \\
+\infty, & \text { otherwise }\end{cases}
\end{aligned}
$$

$\mathcal{F}:=\left\{f_{1}, f_{2}\right\}, f:=\min \left\{f_{1}, f_{2}\right\}$, i.e.,

$$
f: \mathbb{R}^{2} \rightarrow \mathbb{R}:(x, y) \mapsto \max \{-|x|, y\},
$$

and $E:=\{0\} \times \mathbb{R}_{-}$. Then one checks the following:

(1) $\mathcal{F}$ is a compatible system of functions.

(2) $\left\{A_{1}, A_{2}\right\}$ is a compatible system of sets.

(3) For every $(\bar{x}, \bar{y}) \in \mathbb{R}^{2} \backslash E$ with $I_{\mathcal{F}}(\bar{x}, \bar{y})=\{1,2\}$, we must have $(\bar{x}, \bar{y}) \in\{0\} \times \mathbb{R}_{++}$, i.e., $\bar{x}=0$ and $\bar{y}>0$. Then $f(x, y)=y$ locally around $(0, \bar{y})$ and thus

$$
\partial f_{1}(0, \bar{y}) \cap \partial f_{2}(0, \bar{y}) \supseteq \partial f(0, \bar{y})=\{(0,1)\} .
$$

So, all assumptions in Theorems 5.1 and 5.5 are satisfied except that $E$ is infinite. However, for $(0, \bar{y}) \in$ $E \backslash\{(0,0)\}$, we have $\bar{y}<0$; thus, $f_{1}(x, y)=-x+\iota_{A_{1}}(x, y)$ locally around $(0, \bar{y})$. It follows that

$$
\left.\left.\partial f_{1}(0, \bar{y})=(-1,0)+N_{A_{1}}(0, \bar{y})=\right]-\infty,-1\right] \times\{0\}
$$

and similarly that

$$
\partial f_{2}(0, \bar{y})=(1,0)+N_{A_{2}}(x, y)=[1,+\infty[\times\{0\} .
$$

Hence $\partial f_{1}(0, y) \cap \partial f_{2}(0, y)=\varnothing$ and so $f$ is not convex by applying Theorem 7.1 or by direct inspection.

In the previous example, the set $E$ was infinite, but unbounded. In the next (slightly more involved) example, we provide a case where $E$ is bounded.

Example 7.3. Suppose that $X=\mathbb{R}^{2}$, set $I:=\{1, \ldots, 6\}$,

$$
\begin{aligned}
A_{1} & :=\left\{(x, y) \in \mathbb{R}^{2}|| x|+| y \mid \geq 1, x \geq 0, y \geq 0\right\}, \\
A_{2} & :=\left\{(x, y) \in \mathbb{R}^{2}|| x|+| y \mid \geq 1, x \leq 0, y \geq 0\right\}, \\
A_{3} & :=\left\{(x, y) \in \mathbb{R}^{2}|| x|+| y \mid \geq 1, x \leq 0, y \leq 0\right\}, \\
A_{4} & :=\left\{(x, y) \in \mathbb{R}^{2}|| x|+| y \mid \geq 1, x \geq 0, y \leq 0\right\}, \\
A_{5} & :=\left\{(x, y) \in \mathbb{R}^{2}|| x|+| y \mid \leq 1, x \geq 0\right\}, \\
A_{6} & :=\left\{(x, y) \in \mathbb{R}^{2}|| x|+| y \mid \leq 1, x \leq 0\right\}, \\
& f: \mathbb{R}^{2} \rightarrow \mathbb{R}:(x, y) \mapsto \max \{1-|x|,|y|\}, \\
\mathcal{F} & :=\left\{f_{i}\right\}_{i \in I}, \quad \text { where }(\forall i \in I) \quad f_{i}:=f+\iota_{A_{i}},
\end{aligned}
$$


and $E:=(\{0\} \times[-1,1]) \cup\{( \pm 1,0)\}$. Then one checks the following:

(1) Each $f_{i}$ is convex and continuous on $\mathrm{D}_{f_{i}}$ because

$$
\begin{aligned}
& f_{i}(x, y)=y+\iota_{A_{i}}(x, y) \quad \text { for } \quad i \in\{1,2\} \\
& f_{i}(x, y)=-y+\iota_{A_{i}}(x, y) \text { for } \quad i \in\{3,4\} \\
& f_{5}(x, y)=-x+1+\iota_{A_{5}}(x, y) ; \quad \text { and } \\
& f_{6}(x, y)=x+1+\iota_{A_{6}}(x, y) .
\end{aligned}
$$

Consequently, $\mathcal{F}$ is a compatible system of functions.

(2) $\left\{A_{i}\right\}_{i \in I}$ is a compatible system of sets.

(3) $f$ is the piecewise-defined function associated with $\mathcal{F}$.

(4) Take $(\bar{x}, \bar{y}) \in \mathbb{R}^{2} \backslash E$ with $\operatorname{card} I_{\mathcal{F}}(\bar{x}, \bar{y}) \geq 2$. Then

$$
I_{\mathcal{F}}(\bar{x}, \bar{y}) \in\{\{1,2\},\{1,4\},\{1,5\},\{2,3\},\{2,6\},\{3,4\},\{3,6\},\{4,5\}\} .
$$

Suppose, for instance, that $I_{\mathcal{F}}(\bar{x}, \bar{y})=\{1,5\}$. Then $\bar{x}>0, \bar{y}>0$, and $\bar{x}+\bar{y}=1$. We have

$$
\partial f_{1}(\bar{x}, \bar{y})=(0,1)+N_{A_{1}}(\bar{x}, \bar{y})=(0,1)+\mathbb{R}_{+}(-1,-1)
$$

and

$$
\partial f_{5}(\bar{x}, \bar{y})=(0,1)+N_{A_{5}}(\bar{x}, \bar{y})=(-1,0)+\mathbb{R}_{+}(1,1) .
$$

Then

$$
\left(-\frac{1}{2}, \frac{1}{2}\right) \in \partial f_{1}(\bar{x}, \bar{y}) \cap \partial f_{5}(\bar{x}, \bar{y})
$$

similarly, one obtains nonemptiness for the other cases.

We observe that all assumptions in Theorems 5.1 and 5.5 are satisfied except that $E$ is infinite and bounded. However, for every $(0, \bar{y}) \in\{0\} \times]-1,1\left[\subseteq E\right.$, we have $f(x, y)=\min \left\{f_{5}(x, y), f_{6}(x, y)\right\}=-|x|+1$ locally around $(0, \bar{y})$. Clearly, $f$ is not convex.

Acknowledgements. The authors thank the referees for their careful reading and constructive comments. We also thank Rafal Goebel and Gunther Reißig for pointing us to [9,12,17], and to [18], respectively.

\section{REFERENCES}

[1] H.H. Bauschke and P.L. Combettes, Convex Analysis and Monotone Operator Theory in Hilbert Spaces. Springer (2011).

[2] W. Dahmen, Convexity and Bernstein-Bézier polynomials, in Curves and surfaces (Chamonix-Mont-Blanc, 1990). Academic Press (1991) 107-134.

[3] B. Gardiner and Y. Lucet, Computing the conjugate of convex piecewise linear-quadratic bivariate functions. Math. Program. (Series B) 139 (2013) 161-184.

[4] B. Gardiner, K. Jakee and Y. Lucet, Computing the partial conjugate of convex piecewise linear-quadratic bivariate functions. Comput. Optim. Appl. 58 (2014) 249-272.

[5] T.A. Grandine, On convexity of piecewise polynomial functions on triangulations. Comput. Aid. Geom. Des. 6 (1989) $181-187$.

[6] A. Li, Convexity preserving interpolation. Comput. Aid. Geom. Des. 16 (1999) 127-147.

[7] Y. Lucet, What shape is your conjugate? A survey of computational convex analysis and its applications. SIAM Rev. 52 (2010) $505-542$.

[8] Y. Lucet, Techniques and Open Questions in Computational Convex Analysis, in Comput. Anal. Math. Springer (2013) 485500.

[9] M.V. Mihai and C.P. Niculescu, A simple proof of the Jensen-type inequality of Fink and Jodeit. Mediter. J. Math. 13 (2016) $119-126$.

[10] B.S. Mordukhovich and N.M. Nam, An Easy Path to Convex Analysis and Applications. Morgan \& Claypool (2014).

[11] C.P. Niculescu and I. Rovenţa, Relative convexity and its applications. Aequationes Math. 89 (2015) 1389-1400.

[12] A.M. Oberman, The convex envelope is the solution of a nonlinear obstacle problem. Proc. of the AMS 135 (2007) $1689-1694$. 
[13] R.T. Rockafellar, Convex Analysis. Princeton University Press (1970).

[14] R.T. Rockafellar and R.J.-B. Wets, Variational Analysis. Springer (1998).

[15] L.L. Schumaker and H. Speleers, Convexity preserving splines over triangulations. Computer Aid. Geom. Des. 28 (2011) 270-284.

[16] L.L. Schumaker and H. Speleers, Convexity preserving $C^{0}$ splines. Adv. Comput. Math. 40 (2014) 117-135.

[17] J. Sun, On the structure of convex piecewise quadratic functions. J. Optim. Theor. Appl. 72 (1992) 499-510.

[18] A. Weber and G. Reissig, Classical and strong convexity of sublevel sets and application to attainable sets of nonlinear systems. SIAM J. Control Optim. 52 (2014) 2857-2876.

[19] C. Zălinescu, Convex Analysis in General Vector Spaces. World Scientific (2002). 